\title{
PROTOCOL FOR AUTOMATED CONTENT ANALYSIS OF CORPUS TO DETERMINE INFORMATICS COMPETENCIES AMONGST HEALTH SERVICE MANAGERS
}

\author{
Mark Brommeyer1, Mark Mackay2, Zhanming Liang33, Peter Balan² \\ 1. Flinders University, Adelaide, Australia \\ 2. University of South Australia, Adelaide, Australia \\ 3. James Cook University, Townsville, Queensland, Australia
}

Correspondence: mark@brommeyer.com

\begin{abstract}
BACKGROUND:

Competencies have emerged as being important to develop health professionals, including managers in healthcare. Professional institutions adopted specific competency frameworks to guide designing professional development opportunities for health service managers, in particularly managers working in the area of health informatics. The fastgrowing nature of healthcare settings means that the required competencies continue to evolve.
\end{abstract}

OBJECTIVE:

The aim of this protocol is to outline a reflexive thematic analysis process, including using an automated content analysis approach and identify what is missing in existing health service management competency empirical studies in relation to health informatics competencies.

METHODS:

A rapid literature review has been performed using a PRISMA approach for eligibility screening, with 185 publications meeting the inclusion criteria. The Leximancer natural language processing software was used to transform a large corpus of literature from natural language into semantic themes and concepts. A reflexive thematic analysis was then undertaken using the text mining automated content analysis approach to identify predominant concepts and the co-occurrence between them.

RESULTS:

A search strategy was developed using three primary electronic databases: 1) Scopus; 2) ProQuest; and 3) the Cumulative Index to Nursing and Allied Health Literature (CINAHL); and five secondary electronic databases: 1) Web of Science 2) PubMed; 3) ACM Digital Library; 4) Open Access Theses and Dissertations Database; and 5) Google Scholar. The initial search undertaken on 10 November 2020 resulted in 1,212 publications. The results of the reflexive thematic analysis will be submitted for publication by November 2021.

CONCLUSIONS:

New understanding and knowledge in the area of health management competencies, specifically relating to informatics will be developed. Health informatics competencies will be defined for Australian health service managers. Further, this study helps inform the discourse regarding automated content analysis for the healthcare and informatics industry, healthcare organisations and university course requirements. 


\section{KEYWORDS}

Healthcare Management, Health Informatics, Competency, Reflexive Thematic Analysis, Automated Content Analysis

\section{INTRODUCTION}

The Fourth Industrial Revolution is upon us, and it is recognised that it is broad and is characterised by a fusion of technologies across our physical, digital and biological worlds. [1] In healthcare, this is having profound, transformational effects in the diagnosis, delivery and management of care due to the rapid speed of advances in genomic mapping, synthetic biology, nanotechnology engineering, big data, artificial intelligence and robotic automation.

Global forecasts emphasise that these new technologies and innovations have important workforce implications. [1] Firstly, the system needs health service managers who are equipped to lead and manage such transition and health system transformation. Secondly, health service managers need to develop a sound understanding of these new technologies and innovations in order to inform strategic and operational decision-making. Thirdly, there is an imperative that the management capacity of those working in these new fields (e.g., health informaticians) align their management capabilities with those required for the management of health services.

These have far-reaching repercussions for how health service managers are educated and trained, and how management capacity is developed in the emerging and rapidly developing field. Consideration must be given as to how to transform education for both those entering the workforce as well as reskilling the existing workforce. [2] It is imperative to align these required capabilities with the inexorable march of new technologies and labour force trends.

Competencies for health service managers have been established by professional bodies, for example, the Australasian College of Health Service Management (ACHSM) [3] and researched by academics, including Liang, et al. [4] The range of competencies required by health service managers continues to evolve as the industry changes. Therefore, regular review of the competencies required to be demonstrated in the management of healthcare is warranted.
A competency framework, together with a certification process, have also been developed for health informaticians by the Australasian Institute of Digital Health (AIDH) to enable health informaticians to have their competencies recognised. [5] A competency is defined as the specification of knowledge and skill, and the application of that knowledge and skill, to the standard of performance expected in the workplace. [6]

The term digital health is becoming increasingly prevalent in the literature and healthcare generally. [7] As digital health competencies evolve, the further development of competencies for health informatics and health service management can contribute to this evolving discourse. The aim of this protocol to identify what is missing in existing health service management competency empirical studies in relation to health informatics competencies. The method is now detailed.

\section{METHODS}

\section{AIM}

This protocol outlines a reflexive thematic analysis process, including using an automated content analysis approach, to identify what is missing in existing health service management competency empirical studies in relation to health informatics competencies.

\section{METHODOLOGY}

A rapid literature review was performed using a PRISMA approach [8] for eligibility screening. A reflexive thematic analysis of selected papers is being undertaken [9] using Leximancer [8] for the text mining automated content analysis approach to identify predominant concepts and the co-occurrence between them. This will then support sense-making of emerging themes for the health informatics competencies that should be adopted by health service managers.

Using a grounded approach, reflexive thematic analysis is being undertaken. [10] Thematic analysis provides the method for systematically identifying, organising, analysing and advancing insight into themes or patterns of meaning across a large corpus of literature. [9] Importantly, reflexive 
thematic analysis allows the researcher to accentuate meaning as contextual or situated, whilst concomitantly undertaking analysis of the text for meaning. [10, 11] Further, the validity of the researcher's subjectivity is not just recognised as being legitimate, but it is also an intrinsic resource, as reflexive thematic analysis enables the active role of the researcher in the knowledge generation process. [10]

There is a dearth of literature regarding application of automated content analysis for large literature synthesis in qualitative research fields across a range of disciplines [1219] and negligible publications specifically identified in the field of health informatics competencies. Leximancer, as an automated content analysis software that performs quantitative content analysis using machine learning, was used to ascertain what the main concepts are in the informatics competencies amongst health service managers corpus and how they relate to each other. [20] Using the reflexive thematic analysis six-phase approach, the text mining automated content analysis approach identifies predominant concepts and the co-occurrence between them. This augments the acquisition of text patterns and key terms, to reveal patterns that were previously unknown, and information with meaning [21] contained in the corpus of literature. This assists in automating and expediting completion of the first three phases of reflexive thematic analysis, as follows:

\begin{tabular}{|l|l|}
\hline PHASE & PROCES \\
\hline Familiarisation & $\begin{array}{l}\text { Moving from data generation to analysis, i.e., appreciating the data, through } \\
\text { automated sematic analysis, using semantic classifiers to discover unseen } \\
\text { patterns and thematic structures in a body of text [22]. }\end{array}$ \\
\hline Generating codes & $\begin{array}{l}\text { Moving to a more in-depth and systematic engagement with the data, i.e., } \\
\text { making sense of the data, by curating a list of terms, filtering out textual noise } \\
\text { and generating clear patterns to extract more valuable insights [21]. }\end{array}$ \\
\hline Constructing themes & $\begin{array}{l}\text { Moving from codes into overarching themes that accurately and coherently } \\
\text { represent the data, i.e., creating a story about the data, through latent } \\
\text { semantic analysis and singular value determination to uncover themes [21]. }\end{array}$ \\
\hline Revising themes & $\begin{array}{l}\text { Moving to candidate themes and reviewing them to see how each theme } \\
\text { relates to the others, i.e., telling the overall story about the data. }\end{array}$ \\
\hline Defining themes & $\begin{array}{l}\text { Moving to clear definitions of each theme by elucidating the essence and } \\
\text { scope of each theme, i.e., what is meaningful about the data. }\end{array}$ \\
\hline Producing the report & $\begin{array}{l}\text { Moving to a final test of how well the themes work, both individually in relation } \\
\text { to the dataset, and overall, i.e., a logical story with sufficient evidence that the } \\
\text { themes are relevant to the data. }\end{array}$ \\
\hline
\end{tabular}

\section{RAPID REVIEW}

The purpose of this rapid review is to: 1) identify existing core health service management competencies and health informatics competencies; and 2) compare and contrast these two sets of competencies to confirm health informatics competencies that have been incorporated into the health service management competencies. The search was limited to the years 2000 to 2020, as the majority

of the management competency studies were conducted in the past 20 years and the purpose of the rapid review was to identify the most up-to-date competencies. Consequently, reviewing studies conducted prior to the year 2000 was considered to be unnecessary.

\section{SEARCH STRATEGY}




\subsection{Logic Grid}

\begin{tabular}{|c|c|c|c|c|}
\hline TYPE & SEARCH TYPE & SEARCH TERMS & & \\
\hline \multirow{7}{*}{$\begin{array}{l}\text { Peer-reviewed } \\
\text { journal articles }\end{array}$} & SCOPUS & Health informatics & Competencies & Health service manager \\
\hline & ProQuest & Digital health & Capability & Health care manager \\
\hline & Web of Science & Electronic health & Proficiency & Health care executive \\
\hline & $\begin{array}{l}\text { ACM Digital } \\
\text { Library }\end{array}$ & & Qualification & Health care administrator \\
\hline & CINAHL & & Certification & Health service leader \\
\hline & PubMed & & Governance & $\begin{array}{l}\text { Health service } \\
\text { administrator }\end{array}$ \\
\hline & $\begin{array}{l}\text { ProQuest } \\
\text { Dissertations }\end{array}$ & & Policy & Health service executive \\
\hline Grey literature & Google Scholar & & & \\
\hline
\end{tabular}

\subsection{Key words}

The keywords used for the search were: 'health informatics', 'digital health', 'electronic health', 'competencies', 'capability', 'proficiency', 'qualification', 'certification', 'health manager', 'health executive' and 'health administrator'.

String: (health informatics OR digital health OR electronic health) AND (healthcare W2 leadership* OR manage* OR administrat* OR executiv*) AND (competenc* OR capabilit* OR proficienc* OR abilit* OR qualif* OR certif*)

Plain English: health informatics $O R$ digital health $O R$ electronic health AND healthcare leadership* OR manage* OR administrat* OR executiv* AND competenc* OR capabilit* OR proficienc* OR abilit* OR qualif* OR certif*

\subsection{Inclusion criteria}

The following inclusion criteria were used for the search:

- Articles published in English

- Articles published from 2000 to 2020, to ensure up-todate literature reflecting the rapidly evolving nature of health informatics, digital health and health service management
- Publications that were peer-reviewed, empirical and from the grey literature, including government white papers and professional institution position papers.

\subsection{Exclusion criteria}

The following exclusion criteria were applied:

- Publications that were not relevant to the health system context

- Publications that were non-empirical studies, pre-prints and working papers

- Absence of health informatics or health service management competencies.

\section{SEARCH METHODS}

A search strategy was developed using three primary electronic databases: 1) Scopus; 2) ProQuest; and 3) the Cumulative Index to Nursing and Allied Health Literature (CINAHL); and four secondary electronic databases: 1) Web of Science 2) PubMed; 3) ACM Digital Library; 4) Open Access Theses and Dissertations Database. The search was not limited to peer-reviewed literature but also included unpublished grey literature relating to informatics competencies for health service managers, searched 
using Google Scholar; this included government white papers and professional institution position papers.

\section{EXTRACTION AND SCREENING}

1. All publications were downloaded to EndNote (X9.3.3) and saved $(n=1,094)$.

2. Existing publications identified through other sources were added $(n=118)$.

3. Total publications were then saved in one EndNote folder $(n=1,212)$.

4. A removal of duplicates using the find duplicates function in EndNote was performed ( $n=223$ ).

5. The combined search results data was extracted by author(s), year of publication, title, abstract and key words, into Microsoft Excel ( $n=989)$.

6. A removal of duplicates, using the duplicate function in Excel, was performed ( $n=48$ ).

\section{PRISMA FLOW DIAGRAM}
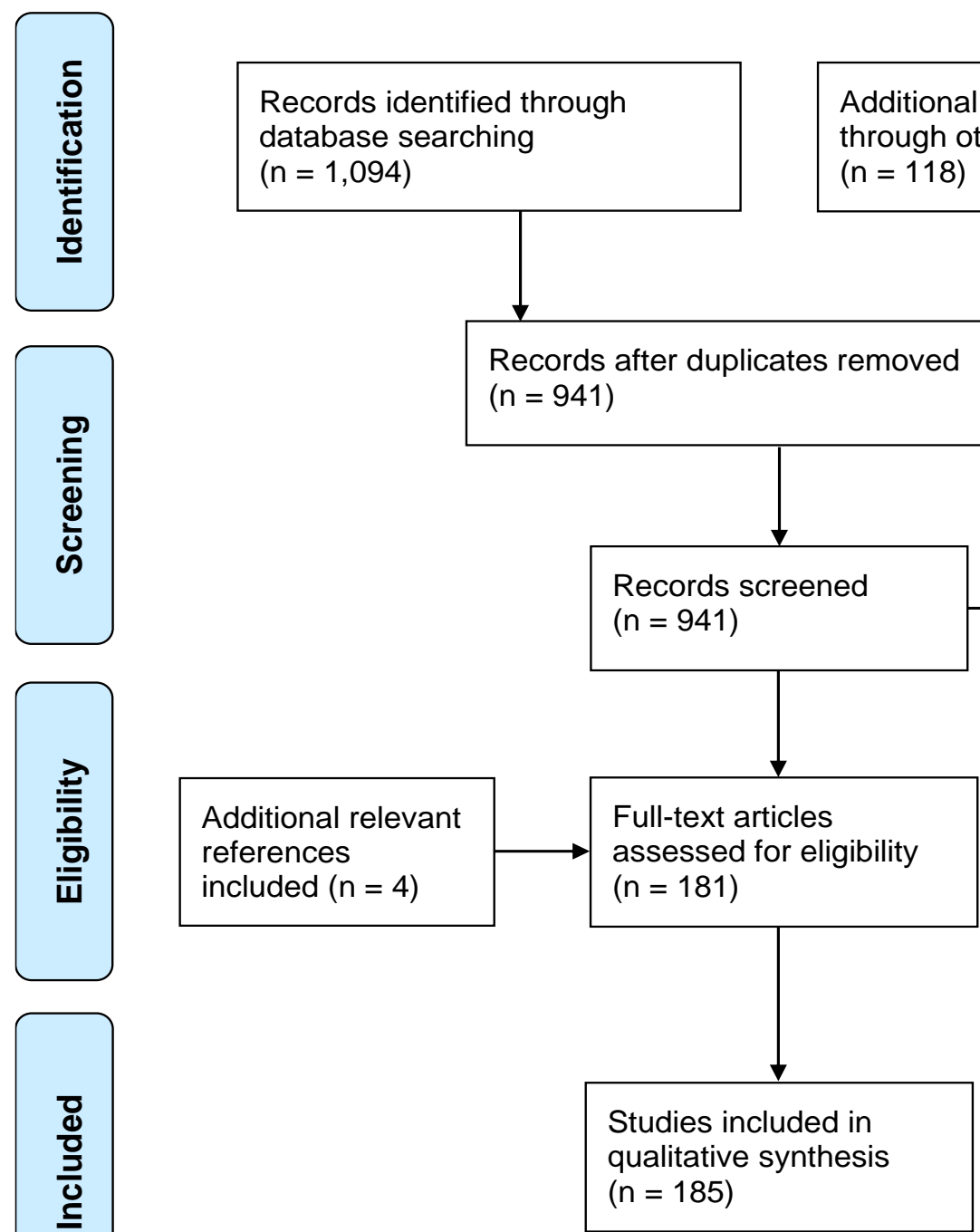

Additional records identified through other sources $(n=118)$ $(n=760)$

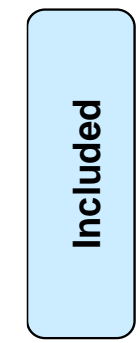

Records after duplicates removed $(\mathrm{n}=941)$ 


\section{DATA SYNTHESIS}

1. The included publications were analysed for applicability, relevance, competencies, context, competence and competency features and characteristics.

2. Reflexive thematic analysis is currently being undertaken to map the themes, including the predominance of the most frequently co-occurring concepts.

3. Following the initial output from the first Leximancer run, concept editing was undertaken to delete labels that were of low semantic meaning and also collapse concept pairs for word capitalisation, pluralisation, and past-tense verbs and prepositions of the same word, that would not impact the analysis and review, e.g. included/including. Based on the semantic and relational extraction algorithms applied, [22] the Concept Map in Figure 1 contained four major themes: 1) health; 2) management; 3) informatics; and 4) electronic; with 31 most frequently co-occurring concepts, as shown in Table 1.

FIGURE 1: CONCEPT MAP REPRESENTING MAJOR THEMES IDENTIFIED FROM THE AUTOMATED CONTENT ANALYSIS WITH "HEALTH", "MANAGEMENT", "INFORMATICS" AND "ElECtRONIC" THEMES HEAT-MAPPED AS (1), (2), (3) AND (4) IN ORDER OF PROMINENCE

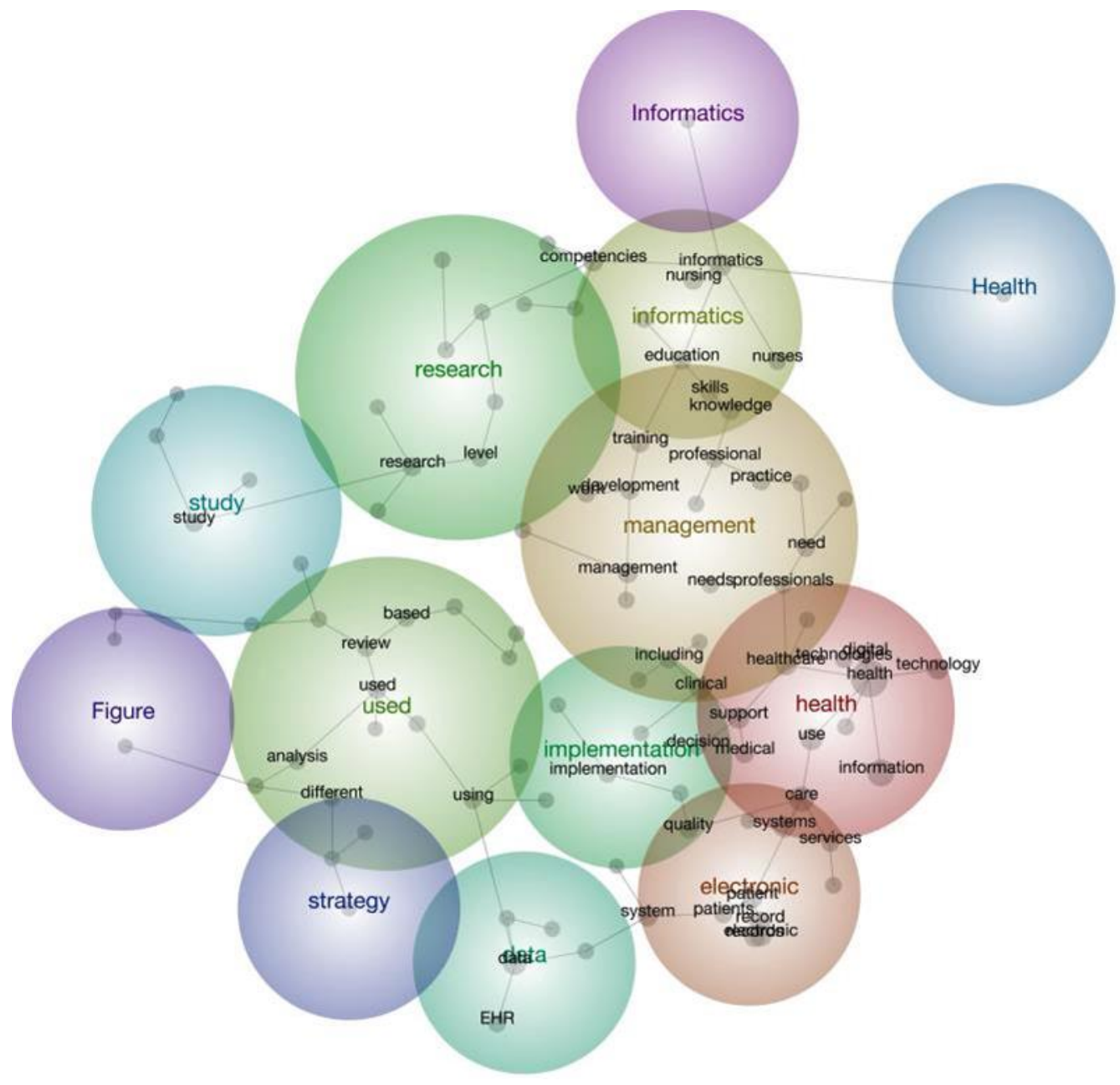

\section{RESULTS}

The first three phases of reflexive thematic analysis have been undertaken with the 185 eligible publications using Leximancer for the text mining, automated content analysis approach to identify predominant concepts and the co- occurrence between them. This has accelerated the progress to completing the final three phases of reflexive thematic analysis (phase 4. revising themes, phase 5 . defining themes, and phase 6. producing the report) to facilitate sense-making sense of and creating a coherence 
about the data. This will inform the logical story of the informatics competencies required for health service managers.
1. INITIAL RESULTS OF THE MOST FREQUENTLY COOCCURRING CONCEPTS BY FREQUENCY

Automated content analysis of the corpus has provided the following results (Table 1, below):

\section{TABLE 1: MOST FREQUENTLY CO-OCCURRING CONCEPTS}

\begin{tabular}{|l|l|l|l|}
\hline \multicolumn{2}{|l|}{ MOST FREQUENTLY CO-OCCURRING CONCEPTS BY FREQUNCY } \\
\hline Health & Management & Informatics & Electronic \\
\hline Information & Practice & Competencies & Record \\
\hline Care & Training & Knowledge & System \\
\hline Healthcare & Development & Nursing & Patient \\
\hline Use & Need & Education & Quality \\
\hline Clinical & Work & Skills & Service \\
\hline Support & Professionals & & \\
\hline Technology & & & \\
\hline Medical & & & \\
\hline Digital & & & \\
\hline Technologies & & & \\
\hline Communication & & & \\
\hline
\end{tabular}

\section{DISCUSSION}

\section{HEALTH INFORMATICS COMPETENCIES FOR HEALTH SERVICE MANAGERS}

A review of the available literature highlighted a paucity in the development of health informatics competencies for health service managers. Globally there has been a wide variety of competency frameworks developed for health service managers. [23-26] There is, however, limited available evidence or agreement of specific health informatics competency requirements for health service managers. [27-29] In Australia, 'the needs of health service organisations have outpaced the science, with organisations adopting competency frameworks for which there is limited evidence and no validated and reliable measurement tools. [30, p.157]

\subsection{Health Informatics Competencies}

Health informatics competencies have been defined by health and informatics organisations in different countries over the last fifty years. Recent development of these competencies has occurred in the United States, United Kingdom and Canada. [5] Collectively, there is a growing consensus that these competency domains include healthcare, biomedical, information and communication technology, information and management science, and human and social context. [5] The United States and the European Union established the EU*US eHealth Work
Project (2016-2018) [31] to develop a structured set of health information technology and informatics implementation competencies, the Health Information Technology Competencies (HITCOMP). One thousand competencies were mapped across five domains including direct patient care, administration, informatics, engineering/information, system/information communication technology and research/biomedicine, against five levels of skill (baseline, basic, intermediate, advanced and expert). The competencies were also mapped to over 250 roles impacted by health information technology, though mainly in the acute care setting. The intermediate, advanced and expert levels in the administration domain, within the Administration/General Management/Governance area of competency, describe nineteen competency statements. These will be analysed for potential incorporation into the confirmation of informatics competencies required for health service managers.

To help more clearly define the body of knowledge underpinning health informatics as a discipline, in Australia, health informatics competencies are considered to be 'the core set of information methods, theories and tools which are relevant to healthcare, biomedical research and public health. However, depending on the application domain, different sub-specialities can be identified'. [5,p.2] By completing the reflexive thematic analysis using the 
overarching themes identified, health informatics competencies required for health service management will be more clearly articulated.

\subsection{Health Service Management Competencies}

Unlike other professions, management is complex and broad in scope, and varies between levels and nature of the positions sitting in different parts of the organisational structure. For a large health service, the management hierarchy usually includes senior, middle and lower levels responsible for setting strategic direction, taking charge of day-to-day operation of the organisation, and service provision respectively. Management literature has confirmed the existence of core competencies for managers despite the differing levels and positions. However, competency is also context sensitive, hence, the required competency level varied between organisation types, levels and positions. [32] In Australia and New Zealand, the Australasian College of Health Service Management provides accreditation to the majority of the University Master of Health Administration Programs, against the management competency framework that they developed. The framework is 'to bring together the combination of competencies and the emphasis within these competencies of the skills, knowledge and attributes necessary for leaders and managers in the health industry'. [3,p.6] These health service management competencies 'are intended to apply across all levels of management and for a range of applications with the College'. [3,p.7]

The empirical evidence supporting effective management as being important to successful operations of healthcare organisations is growing. [30] Furthermore, in an increasing number of countries, healthcare management professional organisations are identifying and certifying health service managers against competency frameworks. [33] It is therefore timely to investigate and illuminate informatics competencies required for health service managers, to augment existing competency frameworks, e.g., ACSHM and AIDH.

\section{EMERGING THEMES}

The overarching themes that are emerging were uncovered through automated content analysis (see Table 1) and the four major themes identified of health, management, informatics and electronic are consistent with the current health service management and informatics discourse regarding the lack of clarity about health informatics competencies for healthcare decision makers [34]. This is further exemplified by the traditional view that a healthcare leader needed competency in clinical services and health management, but now also needs competency in health information technologies [35].

It is well demonstrated that the advantages of text mining include less time and human resources required for analysis, less human bias, along with more reproducible and replicable results for reviewing large corpus of literatures. [21] Further, research across a variety of academic contexts has concluded that when analysing copious data sets, of the various content analysis software technologies available, Leximancer is among the very few that can automate extremely complicated, detailed, and protracted aspects of content analysis. [36] The current study confirms the applicability of Leximancer in developing preliminary understanding of a broad concept, building the foundation of deeper learning and complex analysis.

\section{FUTURE RESEARCH}

In the next stage of this study, phase 4 , the most frequently co-occurring concepts identified will be analysed, using reflexive thematic analysis to see how each theme relates to the others. This will be followed by analysis of defining themes and then production of the final report, to progress the narrative inquiry in support of the informatics competencies required for health service managers, as discussed below.

\section{CONCLUSION}

With the ever-increasing cost challenges faced in the provision of healthcare, for an ageing population with a growing burden of chronic and complex needs [37], it is imperative that our health service management workforce is equipped with the dynamic knowledge, skills, values, and competencies required to make evidence-informed business decisions.

The first stage of this research confirmed the general interest in studying competencies in the field of health service management and health informatics. A reflexive thematic analysis conducted on the 185 articles identified, confirmed that using Leximancer to automate content analysis for identification of predominant concepts and the co-occurrence between them is suitable for conceptualising the health informatics competencies required for health service managers. 


\section{ACKNOWLEDGEMENT}

With grateful acknowledgment to the Australian Government Research Training Program (RTP) fee offset scholarship.

\section{DECLARATION OF CONFLICTING INTERESTS}

The Authors declare that there is no conflict of interest in preparing this manuscript.

\section{References}

1. World Economic Forum. Global future council on the future of health and healthcare report. Geneva: World Economic Forum; 2019.

2. Topol E. The Topol Review: Preparing the healthcare workforce to deliver the digital future. London: NHS Health Education England; 2019.

3. Australasian College of Health Service Management (ACHSM). Master health service management competency framework. Gladesville (AU): ACHSM; 2016.

4. Liang Z, Short SD, Howard PF, Brown CR. Centralised control and devolved responsibilities: personal experiences of senior health executives on the implementation of the area health management model in New South Wales, 1990-1999. Asia Pac J Health Manag 2016; 1: 44-50.

5. Health Informatics Society of Australia (HISA). Certified health informatician Australasia competencies framework. Melbourne: HISA; 2013.

6. Martin-Sanchez F, Rowlands D, Schaper L, Hansen D. The Australian health informatics competencies framework and its role in the Certified Health Informatician Australasia (CHIA) program. Stud Health Technol Inform 2017; 245: 783-787.

7. Brice S, Almond H. Health professional digital capabilities frameworks: a scoping review. J Multidiscip Healthc 2020; 13: 1375-1390.

8. Liberati A, Altman DG, Tetzlaff J, Mulrow C, Gøtzsche PC, loannidis JP, Clarke M, Devereaux PJ, Kleijnen J, Moher D. The PRISMA statement for reporting systematic reviews and meta-analyses of studies that evaluate health care interventions: explanation and elaboration. J Clin Epidemiol 2009; 62(10): 1-34.

9. Braun V, Clarke V. Thematic analysis. In: APA handbook of research methods in psychology. Vol 2. American Psychological Association (APA). Washington: APA; 2012: 57-71.
10. Braun V, Clarke V, Hayfield N, Terry G. Thematic Analysis. In Liamputtong P, editor. Handbook of research methods in health social sciences. Singapore: Springer; 2019: 843-860.

11. Loffe H, Yardley L. Content and thematic analysis. In: Marks DF, Yardley L, editors. Research methods for clinical and health psychology. 1st ed. London: Sage Publications; 2004: 56-69.

12. Smith $A E$, Humphreys MS. Evaluation of unsupervised semantic mapping of natural language with Leximancer concept mapping. Behav Res Methods 2006; 38(2): 262-279.

13. Nunez-Mir GC, lannone BV, Curtis K, Fei S. Evaluating the evolution of forest restoration research in a changing world: a "big literature" review. New For 2015; 46(5): 669-682.

14. Lin $\mathrm{X}$, Zhang $\mathrm{H}$, Wu H, Cui D. Mapping the knowledge development and frontier areas of public risk governance research. Int J Disaster Risk Reduct 2020; 43: 1-12.

15. Hyndman B, Pill S. What's in a concept? A Leximancer text mining analysis of physical literacy across the international literature. Eur Phys Educ Rev 2018; 24(3): 292-313.

16. Thomas DA. Searching for significance in unstructured data: text mining with Leximancer. Eur J Educ Res 2014; 13(2): 235-256.

17. Cheng M, Edwards D. A comparative automated content analysis approach on the review of the sharing economy discourse in tourism and hospitality. Curr Issues Tour 2019; 22(1): 35-49.

18. Cretchley J, Rooney D, Gallois C. Mapping a 40-year history with Leximancer-themes and concepts in the Journal of Cross-Cultural Psychology. J Cross-Cult Psychol 2010; 41 (3): 318-328.

19. Randhawa K, Wilden R, Hohberger J. A bibliometric review of open innovation: setting a research agenda. J Prod Innov Manag 2016; 33(6): 750-772.

20. Ward V, West R, Smith S, McDermott S, Keen J, Pawson $R$, House A. Leximancer analysis. In: The role of informal networks in creating knowledge among health-care managers: a prospective case study 2014 May. NIHR Journals Library.

21. Zengul FD, Oner N, O'Connor SJ, Borkowski N. A text mining study of topics and trends in health care management journals: 1998-2018. Health Care Manage Rev 2021; 2 . 
22. Smith AE, Humphreys MS. Evaluation of unsupervised semantic mapping of natural language with Leximancer concept mapping. Behav Res Methods 2006; 38(2): 264.

23. Calhoun JG, Dollett L, Sinioris ME, Waini JA, Butler PW, Griffith JR, Warden GL. Development of an interprofessional competency model for healthcare leadership. J Healthc Manag 2008; 53(6): 375-389.

24. Garman A, Scribner L. Leading for quality in healthcare: Development and validation of a competency model. J Healthc Manag 201 1; 56(6): 373-384

25. Stefl ME. Common competencies for all healthcare managers: the healthcare leadership alliance model. J Healthc Manag 2008; 53(6): 360-374.

26. Hernandez SR, O'Connor SJ, Meese KA. Global efforts to professionalize the healthcare management workforce: the role of competencies. J Health Admin Educ 2018; 35(2): 157.

27. Australian Digital Health Agency (ADHA). National digital health workforce and education roadmap. Sydney: ADHA; 2020.

28. Butler-Henderson K, Gray K, Pearce C, Ritchie A, Brophy J, Schaper LK, Bennett V, Ryan A. Exploring the health informatics occupational group in the 2018 Australian health information workforce census. Stud Health Technol Inform 2019; 266: 44-50.

29. Health Education England (HEE). NHS Topol Review: preparing the healthcare workforce to deliver the digital future. London: HEE; 2019.

30. Liang Z, Howard PF, Leggat S, Bartram T. Development and validation of health service management competencies. J Health Organ Manag 2018; 32(2): 157-175

31. EU-US eHealth Collaboration Workforce Development Workgroup. Health Information Technology Competencies (HITCOMP) Tool, European Commission's Directorate General for Communication and United States Office of the National Coordinator of Health Information Technology 2018. Available: <http://hitcomp.org/about/> (Accessed 09/08/21)

32. Epstein RM, Hundert EM. Defining and assessing professional competence. J Am Med Assoc 2002; 287(2): 226-235.

33. Howard PF, Liang Z, Leggat S, Karimi L. Validation of a management competency assessment tool for health service managers. J Health Organ Manag 2018; 32(1): 113-134.

34. Hübner U, Thye J, Shaw T, Elias B, Egbert N, Saranto K, Babitsch B, Procter P, Ball MJ. Towards the tiger international framework for recommendations of core competencies in health informatics 2.0: extending the scope and the roles. In MEDINFO 2019: Health and Wellbeing e-Networks for All 2019; 1218-1222.

35. Laukka E, Huhtakangas $M$, Heponiemi T, Kanste $O$. Identifying the roles of healthcare leaders in HIT implementation: A scoping review of the quantitative and qualitative evidence. Int J Environ Res Public Health 2020; 17(8): 1-15.

36. Thomas DA. Searching for significance in unstructured data: text mining with Leximancer. Eur. Educ. Res. J. 2014; 13(2): 235-256.

37. McPake B, Mahal A. Addressing the needs of an aging population in the health system: the Australian case. Health Syst Reform 2017; 3(3): 236-247. 